M. Koike

Nagoya Math. J.

Vol. 89 (1983), 89-107

\title{
A NOTE ON MODULAR FORMS MOD $p$
}

\author{
MASAO KOIKE
}

\section{§1. Introduction}

In this paper we shall study relations among the spaces of modular forms $\bmod p$ attached to $S_{k}(N p, \psi \chi)$ and $S_{k^{\prime}}(N, \psi)$ by using certain identities between dimensions of these spaces.

Let $N$ be a positive integer and $\chi$ be an arbitrary character of $(Z / N Z)^{\times}$. Let $\Gamma_{0}(N)=\left\{\left(\begin{array}{ll}a & b \\ c & d\end{array}\right) \in S L_{2}(Z) \mid c \equiv 0(\bmod N)\right\}$. Let $f(z)$ be a cusp form of weight $k$ satisfying

$$
f(\sigma(z))=(c z+d)^{k} \chi(d) f(z) \quad \text { for all } \sigma=\left(\begin{array}{ll}
a & b \\
c & d
\end{array}\right) \in \Gamma_{0}(N) .
$$

Then we call $f(z)$ a cusp form of type $(k, \chi)$ on $\Gamma_{0}(N)$, and we denote by $S_{k}(N, \chi)$ the space of all cusp forms of type $(k, \chi)$ on $\Gamma_{0}(N)$. If $\chi$ is trivial, we simply denote $S_{k}(N)$.

From now we fix a rational prime $p, p \geq 5$. Let $N$ be a positive integer such that $(p, N)=1$. Let $\psi$ and $\chi$ be arbitrary characters of $(Z / N Z)^{\times}$and $(Z / p Z)^{\times}$respectively such that

$$
\psi \chi(-1)=1
$$

Let $t$ denote the order of $\chi$ and put

$$
\kappa=\frac{(p-1)(t-a)}{t}
$$

with any integer $a$ such that $1 \leq a \leq t,(a, t)=1$. Let $k$ be any even positive integer. Then we shall prove the following simple identities between dimensions of spaces of cusp forms by using Hijikata's explicit trace formula [2]:

THEOREM 1.1. The notation being as above, we have

Received June 1, 1981. 


$$
\begin{aligned}
\operatorname{dim}_{C} S_{k}(N p, \psi \chi)= & \operatorname{dim}_{C} S_{(p+1)(k / 2)-\alpha}(N, \psi) \\
& +\operatorname{dim}_{C} S_{(p+1)(k / 2)-(p-1-\kappa)}(N, \psi) .
\end{aligned}
$$

In $\S 3$, we shall prove a statement analogous to Theorem 1.1 when $k$ is odd, $k \geq 3$.

Let $\widetilde{S_{k}(1)}$ and $\widetilde{S_{k}(p)}$ denote the space of modular forms $\bmod p$ attached to $S_{k}(1)$ and $S_{k}(p)$ respectively. Some of fundamental results on modular forms $\bmod p$, due to Serre and Swinnerton-Dyer are the followings:

$$
\begin{aligned}
& \widetilde{S_{k}(1)} \subset \widetilde{S_{k+p-1}(1)}, \\
& \widetilde{S_{2}(p)}=\widetilde{S_{p+1}(1)} .
\end{aligned}
$$

It is essential to prove (1.5) that it holds

$$
\operatorname{dim}_{C} S_{2}(p)=\operatorname{dim}_{C} S_{p+1}(1)
$$

which is a special case of Theorem 1.1. Hence next to do is to generalize (1.5), namely to get relations between the spaces of modular forms $\bmod p$ attached to $S_{k}(N p, \psi \chi)$ and $S_{k^{\prime}}(N, \psi)$ as an application of Theorem 1.1.

To state further results, we fix $N, \psi$ and $k$ as above. Take an algebraic number field $K$ of finite degree over the rational number field which contains all eigenvalues of all Hecke operators acting on $S_{k}(N p, \psi \chi)$ for all characters $\chi$ of $(Z / p Z)^{\times}$and on $S_{k^{\prime}}(N, \psi)$ for all $k^{\prime} \leq(k / 2)(p+1)$, and all $p(p-1)$-th roots of unity. We fix a prime divisor $\mathfrak{p}$ of $K$ lying over $p$. Let $\nu$ be the valuation of $K$ attached to $p$ normalized by $\nu(p)=p^{-1}$. Also we write $\mathfrak{o}=\{\alpha \in K \mid \nu(\alpha) \leq 1\}$ and $F=\mathfrak{o} / \mathfrak{p}$.

For any character $\chi$ of $(Z / p Z)^{\times}$, we define $V_{\chi}$ and $V_{k^{\prime}}$ by

$$
\begin{aligned}
& V_{\chi}=\left\{f(z)=\sum_{n=1}^{\infty} a_{n} q^{n} \in S_{k}(N p, \psi \chi) \mid a_{n} \in K \text { for all } n \geq 1\right\}, \\
& V_{k^{\prime}}=\left\{g(z)=\sum_{n=1}^{\infty} b_{n} q^{n} \in S_{k^{\prime}}(N, \psi) \mid b_{n} \in K \text { for all } n \geq 1\right\} .
\end{aligned}
$$

where $q=e^{2 \pi i z}$. Then $V_{z}$ and $V_{k^{\prime}}$ are vector spaces over $K$ with same dimensions as those of $S_{k}(N p, \psi \chi)$ and $S_{k^{\prime}}(N, \psi)$ over the complex number field respectively. For any subspace $V$ of $V_{x}$ or $V_{k^{\prime}}$, we define

$$
V(\mathfrak{o})=\left\{f(z)=\sum_{n=1}^{\infty} a_{n} q^{n} \in V \mid a_{n} \in \mathfrak{o} \text { for all } n \geq 1\right\} .
$$

For any $f=\sum_{n=1}^{\infty} a_{n} q^{n}$ in $V(\mathfrak{o})$, we put 


$$
\tilde{f}=\sum_{n=1}^{\infty} \bar{a}_{n} q^{n} \in F[[q]]
$$

where $\bar{a}_{n}=a_{n} \bmod \mathfrak{p}$. $\tilde{f}$ is a cusp form $\bmod p$ in slightly more generalized sense than that of Serre and Swinnerton-Dyer. Let $\tilde{V}$ denote the space over $F$ spanned by $\tilde{f}$ for all $f \in V(\mathfrak{D})$. Then $\tilde{V}$ has the same dimension over $F$ as that of $V$ over $K . \quad \tilde{V}$ is called the space of modular forms $\bmod p$ attached to $V$.

Let $W_{p}=\left(\begin{array}{ll}p x, & 1 \\ p N y, & p\end{array}\right)$ with some rational integers $x$ and $y$, be a matrix with determinant $p$. We define a linear operator $W_{p}$ on $V_{x}$ by

$$
\left(f \mid W_{p}\right)(z)=p^{k / 2}(p N y z+p)^{-k} f\left(\frac{p x z+1}{p N y z+p}\right) .
$$

Then we shall prove that $W_{p}$ gives an isomorphism between $V_{\chi}$ and $V_{\bar{\chi}}$ where $\bar{\chi}$ is the character of $(Z / p Z)^{\times}$defined by $\bar{\chi}(n)=$ the complex conjugate of $\chi(n)$.

Since we fix a prime divisor $\mathfrak{p}$, there exists a unique character $\omega$ of $(Z / p Z)^{\times}$such that

$$
\omega(n) \equiv n \bmod \mathfrak{p}
$$

for all integers $n \in Z,(n, p)=1$. Since $\chi$ is a character of $(Z / p Z)^{\times}$of order $t$, there exists a unique rational integer $a,(1 \leq a \leq t,(a, t)=1)$ such that

$$
\chi=\omega^{(p-1)(t-a) / t} .
$$

Put

$$
\kappa=(p-1)(t-a) / t .
$$

TheOREm 1.2. The notation being as above, there is a decomposition of $V_{x}$ into a direct sum of subspaces $V_{1, x}$ and $V_{2, x}$ satisfying following properties:

$$
\begin{gathered}
\operatorname{dim}_{K} V_{1, \chi}=\operatorname{dim}_{C} S_{(p+1)(k / 2)-(p-1-\kappa)}(N, \psi), \\
\operatorname{dim}_{K} V_{2, \chi}=\operatorname{dim}_{C} S_{(p+1)(k / 2)-\kappa}(N, \psi) . \\
\widetilde{V_{1, \chi}}=\widetilde{V_{(p+1)(k / 2)-(p-1-\kappa)}} . \\
\left(\overparen{\left.V_{2, \chi} \mid W_{p}\right)=\widetilde{V_{(p+1)(k / 2)-\kappa}}} .\right.
\end{gathered}
$$

When $N=1, k=2$ and $\chi$ is trivial, Theorem 1.2 implies $\widetilde{S_{2}(p)}=\widetilde{S_{p+1}}(1)$.

As a corollary of Theorem 1.2, we shall prove 
Corollary 1.3. For any Hecke operator $T(n)$ with $(n, N p)=1$, the following congruence holds:

$$
\begin{aligned}
\operatorname{tr} T(n) \text { on } S_{k}(N p, \psi \chi) \equiv & \operatorname{tr} T(n) \text { on } S_{(p+1)(k / 2)-(p-1-k)}(N, \psi) \\
& +n^{\kappa} \operatorname{tr} T(n) \quad \text { on } S_{(p+1)(k / 2)-\kappa}(N, \psi) \quad(\bmod \mathfrak{p}) .
\end{aligned}
$$

Some results of this note were already announced in [5] without proofs. For general terminology on automorphic forms, we refer to Shimura's textbook [9].

When $k=2$ and $\psi$ and $\chi$ are trivial characters, Dr. Hatada obtained earlier similar results to Theorem 1.1 and Theorem 1.2 in Part 2 of his doctoral thesis at University of Tokyo, April 1979.

\section{§2. Proof of Theorem 1.1}

We shall prove Theorem 1.1.

Hijikata's trace formula for $\operatorname{dim}_{C} S_{k}(N, \chi)$ can be read as follows [2]: let $N$ be a positive integer and $k \geq 2$ be an integer. Let $\chi$ be an arbitrary $C^{\times}$-valued character of $(Z / N Z)^{\times}$such that $\chi(-1)=(-1)^{k}$.

Then we have

$$
\begin{aligned}
\operatorname{dim}_{C} S_{k}(N, \chi)= & t_{v}(k, N, \chi)+t_{p}(k, N, \chi)+t_{e 1}(k, N, \chi) \\
& +t_{e 2}(k, N, \chi)+t_{\delta}(k, N, \chi),
\end{aligned}
$$

where

$$
\begin{aligned}
& t_{v}(k, N, \chi)=\frac{k-1}{12} \prod_{p \mid N} p^{\nu}\left(1+\frac{1}{p}\right), \\
& t_{e 1}(k, N, \chi)=\left\{\begin{array}{cc}
-\frac{1}{3} \frac{\omega^{k-1}-\omega^{\prime k-1}}{\omega-\omega^{\prime}} \prod_{p \mid N} \frac{1}{2}\left(1+\left(\frac{-3}{p}\right)\right)\left(\chi_{p}(\omega)+\chi_{p}\left(\omega^{\prime}\right)\right) \\
\text { if } 3^{2} \nmid N, \\
0 & \text { if } 3^{2} \mid N,
\end{array}\right. \\
& t_{e 2}(k, N, \chi)=\left\{\begin{array}{cl}
-\frac{1}{4} i^{k-2} \prod_{p \backslash N} \frac{1}{2}\left(1+\left(\frac{-4}{p}\right)\right)\left(\chi_{p}(i)+\chi_{p}(-i)\right) \\
0 & \text { if } 4 \nmid N, \text { and } k \text { even, } \\
0 & \text { otherwise },
\end{array}\right.
\end{aligned}
$$

$$
\begin{aligned}
& t_{\delta}(k, N, \chi)= \begin{cases}1 & \text { if } k=2 \text { and } \chi \text { is trivial, } \\
0 & \text { otherwise },\end{cases} \\
& \chi=\prod_{P \mid N} \chi_{p}, \quad N=\prod_{P \mid N} p^{\nu}, X^{2}+X+1=(X-\omega)\left(X-\omega^{\prime}\right),
\end{aligned}
$$


and all products run over all prime divisors $p$ of $N . t_{p}(k, N, \chi)$ denotes the term which comes from the contribution of the parabolic elements. Later we shall describe it clearly.

Remark. If $\chi$ does not satisfy the condition $\chi(-1)=(-1)^{k}, \operatorname{dim}_{c} S_{k}(N, \chi)$ is automatically equal to zero. But the right hand side does not vanish generally. Hence (2.1) does not hold in general if $\chi(-1) \neq(-1)^{k}$.

Now we fix a rational prime $p \geq 5$. Let $N$ be a positive rational integer which is prime to $p$. Let $k \geq 2$ be an even rational integer. Let $\psi$ and $\chi$ be arbitrary $C^{\times}$-valued characters of $(Z / N Z)^{\times}$and $(Z / p Z)^{\times}$ respectively satisfying

$$
\psi \chi(-1)=1
$$

Let $t$ denote the order of $\chi$. Then $t$ is a divisor of $p-1$. For any integer $a$ such that $1 \leq a \leq t$ and $(a, t)=1$, we put $\kappa=((p-1)(t-a)) / t$. Put

$$
k_{1}=\frac{k}{2}(p+1)-(p-1-\kappa),
$$

and

$$
k_{2}=\frac{k}{2}(p+1)-\kappa
$$

We shall prove

Theorem 2.1. The notation being as above, we have

$$
t_{\alpha}(k, N p, \psi \chi)=t_{\alpha}\left(k_{1}, N, \psi\right)+t_{\alpha}\left(k_{2}, N, \psi\right)
$$

for $\alpha=v, p, e 1, e 2, \delta$.

Remark 2.2. It is clear that Theorem 1.1 follows from Theorem 2.1.

Proof. We should remark that $\psi(-1)=(-1)^{k_{1}}=(-1)^{k_{2}}$. Because if $\psi(-1)=1$, then $\chi(-1)=1$. Hence $\kappa$ is even. So $k_{1}$ and $k_{2}$ are also even. If $\psi(-1)=-1$, then $\chi(-1)=-1$. Hence $\kappa$ is odd. So $k_{1}$ and $k_{2}$ are also odd. We should remark that, for $\alpha=v, e 1, e 2$, the terms corresponding to prime factors $\ell$ of $N$ are common in both hand sides of (2.6).

For $\alpha=v$, we have 


$$
\begin{aligned}
t_{v}\left(k_{1}, 1, \psi\right)+t_{v}\left(k_{2}, 1, \psi\right) & =\frac{1}{12}\left\{k_{1}+k_{2}-2\right\} \\
& =\frac{1}{12}\{k(p+1)-(p-1)-2\} \\
& =\frac{1}{12}(k-1)(p+1) \\
& =t_{v}(k, p, \chi) .
\end{aligned}
$$

For $\alpha=\delta$, the left hand side of (2.6) is equal to 1 if and only if $k=2$ and $\psi$ and $\chi$ are trivial characters. Then we have $t=1$ and $\kappa=0$. Hence $k_{1}=2$ and $k_{2}=p+1$. Therefore the right hand side of (2.6) is equal to 1. Otherwise $k_{1} \neq 2$ and $k_{2} \neq 2$. Hence the right hand side of (2.6) is equal to zero.

For $\alpha=e 1$, it is sufficient to prove:

$$
\begin{gathered}
\left(\omega^{k-1}-\omega^{\prime k-1}\right) \frac{1}{2}\left(1+\left(\frac{-3}{p}\right)\right)\left(\chi(\omega)+\chi\left(\omega^{\prime}\right)\right) \\
=\omega^{k_{1}-1}-\omega^{\prime k_{1}-1}+\omega^{k_{2}-1}-\omega^{\prime k_{2}-1} .
\end{gathered}
$$

When $p \equiv 2(\bmod 3)$, the left hand side of $(2.6)$ is equal to zero. On the other hand, we have $k_{1} \equiv \kappa-1(\bmod 3)$ and $k_{2} \equiv-\kappa(\bmod 3)$. Hence $\omega^{k_{1}-1}$ $=\omega^{\kappa-2}$ and $\omega^{k_{2}-1}=\omega^{-\kappa-1}$. To prove (2.6), it is sufficient to prove the following lemma:

LemMa 2.3. For any integer $\kappa$, we have

$$
\omega^{x-2}-\omega^{k-2}=\omega^{\prime-x-1}-\omega^{-\kappa-1}
$$

Proof. If $\kappa \equiv 0(\bmod 3)$, we have $\omega^{\kappa-2}=\omega$ and $\omega^{\prime-x-1}=\omega^{\prime-1}=\omega$. If $\kappa \equiv 1(\bmod 3)$, we have $\omega^{\kappa-2}=\omega^{-1}=\omega^{\prime}$ and $\omega^{\prime-x-1}=\omega^{\prime-2}=\omega^{\prime}$. If $\kappa \equiv 2$ $(\bmod 3)$, we have $\omega^{\kappa-2}=1$ and $\omega^{-x-1}=1$. Hence Lemma 2.3 is proved.

When $p \equiv 1(\bmod 3)$, the left hand side of $(2.6)$ is equal to $(\chi(\omega)+$ $\left.\chi\left(\omega^{\prime}\right)\right)\left(\omega^{k-1}-\omega^{\prime k-1}\right)$. In this case, $\omega^{x}$ is proved to be equal to $\chi(\omega)$ or $\chi\left(\omega^{\prime}\right)$. Since $\omega^{k_{1}-1}=\omega^{k} \cdot \omega^{k-1}$ and $\omega^{k_{2}-1}=\omega^{-k} \omega^{k-1}$, we have

$$
\omega^{k_{1}-1}-\omega^{\prime k_{1}-1}+\omega^{k_{2}-1}-\omega^{\prime k_{2}-1}=\left(\chi(\omega)+\chi\left(\omega^{\prime}\right)\right)\left(\omega^{k-1}-\omega^{\prime k-1}\right) .
$$

Hence Theorem 2.1 is proved for $\alpha=e 1$. Now we shall prove (2.6) for $\alpha$ $=e 2$. If $\chi(-1)=-1, \kappa$ is odd. Hence $k_{1}$ and $k_{2}$ are also odd. Hence the both hand sides of (2.6) are equal to zero. Therefore we may assume that $\chi(-1)=1$. Hence $k_{1}$ and $k_{2}$ are even, so it is sufficient to prove: 


$$
\left(1+\left(\frac{-4}{p}\right)\right) \chi(i) i^{k-2}=i^{k_{1}-2}+i^{k_{2}-2} .
$$

When $p \equiv 3(\bmod 4)$, the left hand side of $(2.8)$ is equal to zero. Since $k_{1}-$ $k_{2} \equiv p-1 \equiv 2(\bmod 4)$, we have $i^{k_{1}-2}+i^{k_{2}-2}=0$. Hence $(2.8)$ is true. When $p \equiv 1(\bmod 4)$, the left hand side of $(2.8)$ is equal to $2 \chi(i) i^{k-2}$. Since $\chi(-1)=1, \chi(i)$ is equal to \pm 1 . Hence $\chi(i)=i^{\kappa}=i^{-\kappa}$. Therefore we have $i^{k_{1}-2}=i^{\kappa} i^{k-2}$ and $i^{k_{2}-2}=i^{-\kappa} i^{k-2}=i^{\kappa} \cdot i^{k-2}$, so

$$
i^{k_{1}-2}+i^{k_{2}-2}=2 i^{k} \cdot i^{k-2}=2 \chi(i) i^{k-2} .
$$

Now we calculate $t_{p}(k, N, \chi)$ following the formula in [2]. We use the same notation as in [2]. Let $s=2$ and $\Phi(X)=(X-1)^{2}$. For $1 \leq f \leq N$, we calculate $c(2, f)$. Let $p$ be a prime divisor of $N$ and $\rho=\operatorname{ord}_{p} f$. Then $\nu=$ $\operatorname{ord}_{p} N$. Let $p^{m}$ denote the conductor of $\chi_{p}$. Put

$$
\begin{aligned}
& \tilde{A}=\left\{x \in Z \mid(x-1)^{2} \equiv 0 \bmod p^{\nu+2 \rho}, 2 x \equiv 2 \bmod p^{\rho}\right\} \\
& \tilde{B}=\left\{x \in \tilde{A} \mid(x-1)^{2} \equiv 0 \bmod p^{\nu+1+2 \rho}\right\} .
\end{aligned}
$$

Let $A$ (resp. $B$ ) be a complete system of representatives of $\tilde{A}$ (resp. $\tilde{B}$ ) modulo $p^{\nu+\rho} . c(2, f)$ and $c(2, f, p)$ are defined by the followings:

$$
\begin{aligned}
& c(2, f)=\prod_{p \mid N} c(2, f, p), \\
& c(2, f, p)=\sum_{x \in A} \chi_{p}(x)+\sum_{y \in B} \chi_{p}(2-y) .
\end{aligned}
$$

(Case of $\nu=2 n, n \in Z$ ). We have $\tilde{A}=\left\{x \in Z \mid x \equiv 1 \bmod p^{n+\rho}\right\}$ and $\tilde{B}=$ $\left\{x \in Z \mid x \equiv 1 \bmod p^{n+1+\rho}\right\}$. Hence we have $A=\left\{x \bmod p^{2 n+\rho}, x \equiv 1 \bmod p^{n+\rho}\right\}$ and $B=\left\{x \bmod p^{2 n+\rho}, x \equiv 1 \bmod p^{n+\rho+1}\right\}$, so $|A|=p^{n}$ and $|B|=p^{n-1}$. Therefore we have

$$
c(2, f, p)= \begin{cases}p^{n}+p^{n-1} & \text { if } m \leq n+\rho \\ p^{n-1} & \text { if } m=n+\rho+1 \\ 0 & \text { if } m>n+\rho+1\end{cases}
$$

(Case of $\nu=2 n+1, n \in Z)$. We have $\tilde{A}=\tilde{B}=\left\{x \in Z \mid x \equiv 1 \bmod p^{n+1+\rho}\right\}$. Hence we have $A=B=\left\{x \bmod p^{2 n+1+\rho}, x \equiv 1 \bmod p^{n+1+\rho}\right\}$ and $|A|=|B|=$ $p^{n}$. Therefore we have

$$
c(2, f, p)= \begin{cases}2 p^{n} & \text { if } m \leq n+\rho+1, \\ 0 & \text { if } m>n+\rho+1 .\end{cases}
$$

Since $\chi(-1)=(-1)^{k}, t_{p}(k, N, \chi)$ is given by the following: 


$$
\begin{aligned}
t_{p}(k, N, \chi) & =-\frac{2}{4 N} \sum_{f} c(2, f), \\
& =-\frac{2}{4 N} \sum_{f} \prod_{p \mid N} c(2, f, p),
\end{aligned}
$$

where $f$ ranges from 1 to $N$.

We should write $c(2, f, N, \chi)$ or $c(2, f, p, N, \chi)$ instead of $c(2, f)$ or $c(2, f, p)$ to make explicit contributions of the level $N$ and the character $\chi$. It is clear that $t_{p}(k, N, \chi)$ is independent of $k$.

LEMma 2.4. Let $f$ be an integer such that $1 \leq f \leq N$. Put $f^{\prime}=f+i N$ for $0 \leq i \leq p-1$. Then, for any prime divisor $\ell$ of $N$, we have

$$
c\left(2, f^{\prime}, \ell, N p, \psi \chi\right)=c(2, f, \ell, N, \psi) .
$$

Proof. Put $\rho=\operatorname{ord}_{\ell} f, \rho^{\prime}=\operatorname{ord}_{\ell} f^{\prime}$ and $\nu=\operatorname{ord}_{\ell} N$. Let $\ell^{m}$ denote the conductor of $\psi_{\ell}$. Since $\ell \neq p$, we have $(\psi \chi)_{\ell}=\psi_{\ell}$. If $\rho<\nu, \rho^{\prime}$ is equal to $\rho$. Hence (2.12) is true. If $\rho \geq \nu$, it also holds $\rho^{\prime} \geq \nu$. But it is clear that $m \leq \nu$, so we have

$$
n+\rho \geq m \text { and } n+\rho^{\prime} \geq m .
$$

Hence (2.12) is true.

LEMMA 2.5. If $\nu=1$, we have

$$
c(2, f, p)=2
$$

for any integer $f$.

Proof. When $\nu=1$, we have $n=0$. Hence $n+\rho+1 \geq 1$ and $m \leq 1$, so $n+\rho+1 \geq m$. Therefore $c(2, f, p)=2$.

Now we shall prove Theorem 2.1 for $\alpha=p$. We have

$$
\begin{aligned}
& t_{p}(k, N p, \psi \chi)=-\frac{2}{4 N p} \sum_{\substack{f^{\prime}=f_{i N N} \\
0 \leq i \leq N \leq 1}} c\left(2, f^{\prime}, p, N p, \psi \chi\right) \prod_{\ell|N| N \mid} c\left(2, f^{\prime}, \ell, N p, \psi \chi\right) \\
& =-\frac{4}{4 N p} \sum_{\substack{f^{\prime}=f+i N \\
1 \leq N \leq N \\
0 \leq i \leq p-1}} \prod_{\ell \mid N} c(2, f, \ell, N, \psi) \\
& =-\frac{4}{4 N} \sum_{1 \leq f \leq N} c(2, f, N, \psi) \\
& =t_{p}\left(k_{1},{ }^{\prime} N,,_{-}^{\prime} \psi\right)+t_{p}\left(k_{2},{ }_{-}^{\prime} N, \psi\right) \text {. }
\end{aligned}
$$

This completes the proof. 


\section{§3. Supplements to Theorem 1.1}

Here we shall give several supplements to Theorem 1.1.

Let $S_{k}^{0}(N, \chi)$ denote the subspace of all new forms in $S_{k}(N, \chi)$. We consider the same situation as in Theorem 1.1. Let $M$ denote the conductor of $\psi$. If the conductor of $\chi$ is $p$, we have

$$
S_{k}(N p, \psi \chi)=\oplus_{M} \underset{d \mid(N / M)}{\oplus} S_{k}^{0}(M p, \psi \chi)^{d} \quad \text { (direct sum) }
$$

where $S_{k}^{0}(M p, \psi \chi)^{d}=\left\{f(d z) \mid f \in S_{k}^{0}(M p, \psi \chi)\right\}$ and the summation ranges over all positive divisors $M$ of $N$ which are multiples of $M$ and over all positive divisors $d$ of $N / M$.

If $\chi$ is the trivial character, we have $S_{k}(N p, \psi \chi)=S_{k}(N p, \psi)$ and

$$
S_{k}(N p, \psi)=\oplus_{M} \underset{d \mid(N / M)}{\oplus}\left\{S_{k}^{0}(M, \psi)^{d} \oplus S_{k}^{0}(M, \psi)^{d p}\right\} \underset{M}{\oplus} \underset{d \mid(N / M)}{\oplus} S_{k}^{0}(M p, \psi)^{d}
$$

(direct sum)

where the summation ranges over the same sets as above. Therefore, using inductions on levels and Theorem 1.1, we can easily prove the following:

TheOREM 3.1. We use the same notation as in Theorem 1.1. We assume that $\chi$ is not trivial, then we have

$$
\begin{aligned}
\operatorname{dim}_{C} S_{k}^{0}(N p, \psi \chi)= & \operatorname{dim}_{C} S_{(k / 2)(p+1)-(p-1-x)}^{0}(N, \psi) \\
& +\operatorname{dim}_{C} S_{(k / 2)(p+1)-\kappa}^{0}(N, \psi) .
\end{aligned}
$$

We also have

$$
\begin{aligned}
\operatorname{dim}_{C} S_{k}^{0}(N p, \psi)+2 \operatorname{dim}_{C} S_{k}^{0}(N, \psi)=\operatorname{dim}_{C} S_{(k / 2)(p+1)-(p-1)}^{0}(N, \psi) \\
+\operatorname{dim}_{C} S_{(k / 2)(p+1)}^{0}(N, \psi),
\end{aligned}
$$

where $\psi$ is an arbitrary character of $(Z / N Z)^{\times}$such that $\psi(-1)=1$.

Combining the above theorem and Hijikata's results in [3], we can prove identities between dimensions of spaces of cusp forms with respect to Fuchsian groups which are obtained from split orders of indefinite division quaternion algebras.

In Theorem 1.1, we assume that $k$ is even. Now we consider the case that $k$ is odd and $k \geq 3$. Let $\psi$ and $\chi$ be arbitrary characters of $(Z / N Z)^{\times}$ and $(Z / p Z)^{\times}$respectively satisfying $\psi \chi(-1)=-1$. Let $t$ denote the order of $\chi$. Put $\kappa=((p-1)(t-a)) / t$ with any integer $a$ such that $1 \leq a \leq t$ and $(a, t)=1$. Put 


$$
k_{1}=\frac{k}{2}(p+1)-(p-1-\kappa)
$$

and

$$
k_{2}=\frac{k}{2}(p+1)-\kappa
$$

We have to check whether $\psi(-1)=(-1)^{k_{1}}$ or not.

LEMMA 3.2. We have

$$
\psi(-1)= \begin{cases}(-1)^{k_{1}}=(-1)^{k_{2}} & \text { if } p \equiv 1(\bmod 4), \\ -(-1)^{k_{1}}=-(-1)^{k_{2}} & \text { if } p \equiv 3(\bmod 4) .\end{cases}
$$

Proof. If $p \equiv 3(\bmod 4)$, we have $k_{1} \equiv \kappa(\bmod 2)$ and $k_{2} \equiv \kappa(\bmod 2)$. Since $\chi(-1)=(-1)^{x}$, we have $\psi(-1)=-\chi(-1)=-(-1)^{x}=-(-1)^{k_{1}}=$ $-(-1)^{k_{2}}$. If $p \equiv 1(\bmod 4)$, we have $k_{1} \equiv 1+\kappa$ and $k_{2} \equiv 1+\kappa(\bmod 2)$. Since $\chi(-1)=(-1)^{x}$, we have $\psi(-1)=-\chi(-1)=(-1)^{1+\kappa}=(-1)^{k_{1}}=(-1)^{k_{2}}$.

Considering a statement analogous to Theorem 1.1 in the case $k$ is odd, we have almost no problem if $p \equiv 1(\bmod 4)$. But, if $p \equiv 3(\bmod 4)$, the right hand side of (1.3) is automatically equal to zero, because $\operatorname{dim}_{C} S_{k_{1}}(N, \psi)=0$ if $\psi(-1) \neq(-1)^{k_{1}}$. So we have to define the followings: let $N$ be a positive integer and $k \geq 2$ be an integer. Let $\chi$ be an arbitrary $C^{\times}$-valued character of $(Z / N Z)^{\times}$. We don't assume that $\chi(-1)=(-1)^{k}$. For the triple $(k, N, \chi)$, we define $t_{v}(k, N, \chi), t_{e 1}(k, N, \chi), t_{e 2}(k, N, \chi)$ and $t_{\delta}(k, N, \chi)$ by $(2.2) \sim(2.5)$. For the definition of $t_{p}(k, N, \chi)$, we use (2.11). The definitions of these numbers don't need the condition $\chi(-1)=(-1)^{k}$.

Then we put

$$
d(k, N, \chi)=\sum_{\alpha=v, e 1, e 2, p, \delta} t_{\alpha}(k, N, \chi) .
$$

Hence if $\chi(-1)=(-1)^{k}, d(k, N, \chi)$ coincides with $\operatorname{dim}_{C} S_{k}(N, \chi)$.

Theorem 3.3. We suppose $k$ is odd, $k \geq 3$ and $\psi \chi(-1)=-1$. The notation being as above, we have

$$
d(k, N p, \psi \chi)=d\left(k_{1}, N, \psi\right)+d\left(k_{2}, N, \psi\right) .
$$

Proof. In proving Theorem 2.1, we don't use the condition $k$ is even except for the proof in the case $\alpha=e 2$. Since $k$ is odd, the left hand side is always equal to zero. If $p \equiv 3(\bmod 4)$, we have $(-4 / p)=-1$, so the right hand side is equal to zero. We may assume $p \equiv 1(\bmod 4)$. Then 
we have $k_{1} \equiv k_{2} \equiv 1+\kappa(\bmod 2)$. If $\kappa$ is even, namely $\chi(-1)=1$, the right hand side is equal to zero. If $\chi(-1)=-1$, we have $\chi(i)+\chi(-i)=0$.

This completes the proof.

ExAmple 3.4. We calculate $d(k)=d\left(k, 1, \chi^{0}\right)$ for any odd $k$ and the trivial character $\chi^{0}$. We have

$$
\begin{aligned}
d(k) & =\frac{k-1}{12}-\frac{1}{2}-\frac{1}{3} \frac{\omega^{k-1}-\omega^{\prime k-1}}{\omega-\omega^{\prime}} \\
& = \begin{cases}\frac{m}{2}-\frac{1}{2} & \text { if } k=6 m+1 \\
\frac{m}{2} & \text { if } k=6 m+3, \\
\frac{m}{2}-\frac{1}{2} & \text { if } k=6 m+5 .\end{cases}
\end{aligned}
$$

Let $p$ be a rational prime such that $p \equiv 3(\bmod 4)$ and $p \geq 5$. We apply Theorem 3.3 for $\operatorname{dim}_{c} S_{k}(p, \chi)$ for an odd $k, k \geq 3$ and an arbitrary character $\chi$ of $(Z / p Z)^{\times}$such that $\chi(-1)=-1$. Let $t$ denote the order of $\chi$. Then we have

$$
\begin{aligned}
\operatorname{dim}_{c} S_{k}(p, \chi)= & d\left(\left\{\frac{k}{2}(p+1)-\left(p-1-\frac{p-1}{t}\right)\right\}\right) \\
& +d\left(\left\{\frac{k}{2}(p+1)-\frac{p-1}{t}\right\}\right) .
\end{aligned}
$$

When $\chi(n)=(n / p)$, we have

$$
\operatorname{dim}_{C} S_{k}\left(p,\left(\frac{-}{p}\right)\right)=2 d\left(\left\{\frac{k-1}{2}(p+1)+1\right\}\right)
$$

\section{§4. Proof of Theorem 1.2}

We use the same notation as in the introduction. We prove the following lemma.

Lemma 4.1. $W_{p}$ gives the isomorphism between $V_{\chi}$ and $V_{\bar{\chi}}$.

Proof. By virtue of results of Miyake [7] and Asai [1], $W_{p}$ gives the isomorphism between $S_{k}(N p, \psi \chi)$ and $S_{k}(N p, \psi \bar{\chi})$. Therefore it is sufficient to prove that $W_{p}$ maps $V_{\chi}$ into $V_{\bar{z}}$, or $W_{p}$ maps a suitable basis of $V_{\chi}$ into $V_{\bar{\chi}}$. As a suitable basis of $V_{\chi}$, we can choose following elements:

(i) $f_{i}(z)=\sum_{n=1}^{\infty} a_{n} q^{n} \in S_{k}^{0}(N p, \psi \chi)$ are common eigenfunctions of all 
Hecke operators such that $a_{1}=1$ which are called primitive eigenforms in $S_{k}(N p, \psi \chi)$,

(ii) $g_{j}(z)$ are primitive eigenforms in $S_{k}(M, \psi \chi)$ for any positive divisors $M$ of $N p$ such that $\psi \chi$ is defined modulo $M$,

(iii) $g_{j}(d z)$ for any positive divisors $d$ of $N p / M$.

By Lemma 3 in [1], $W_{p}$ maps $f_{i}(z)$ into $V_{\bar{z}}$. If $g_{j}(z)$ is a primitive eigenform in $S_{k}(M p, \psi \chi)$ where $M$ is a positive divisor of $N$, for any positive divisors $d$ of $N / M$, we have

$$
\begin{aligned}
g_{j}(d z) \mid W_{p} & =d^{-k / 2} g_{j}(z) \mid\left[\begin{array}{ll}
d & 0 \\
0 & 1
\end{array}\right]\left[\begin{array}{ll}
p x, & 1 \\
p N y, & p
\end{array}\right] \\
& =d^{-k / 2} g_{j}(z) \mid\left[\begin{array}{ll}
p x, & d \\
p(N / d) y, & p
\end{array}\right]\left[\begin{array}{ll}
d & 0 \\
0 & 1
\end{array}\right] .
\end{aligned}
$$

Hence we can apply Lemma 3 in [1] to $g_{j}(z)$, so $W_{p}$ maps $g_{j}(d z)$ into $V_{\bar{x}}$. If $g_{j}(z)$ is a primitive eigenform in $S_{k}(M, \psi)$ where $M$ is a positive divisor of $N, \chi$ must be the trivial character. Hence we have, for any positive divisors $d$ of $N / M$,

$$
\begin{aligned}
g_{j}(d z) \mid W_{p} & =d^{-k / 2} g_{j}(z) \mid\left[\begin{array}{ll}
d & 0 \\
0 & 1
\end{array}\right]\left[\begin{array}{ll}
p x, & 1 \\
p N y, & p
\end{array}\right] \\
& =d^{-k / 2} g_{j}(z) \mid\left[\begin{array}{ll}
x, & d \\
(N / d) y, & p
\end{array}\right]\left[\begin{array}{ll}
p d & 0 \\
0 & 1
\end{array}\right] .
\end{aligned}
$$

Since $\left[\begin{array}{ll}x, & d \\ (N / d) y, p\end{array}\right]$ belongs to $\Gamma_{0}(N / d), W_{p}$ maps $g_{j}(d z)$ into $V_{\bar{z}}$. Hence Lemma 4.1 is completely proved.

Let $K_{\mathfrak{p}}$ denote the completion of $K$ at $\mathfrak{p}$ and $\mathfrak{b}_{\mathfrak{p}}$ the closure of $\mathfrak{v}$ in $K_{\mathfrak{p}}$. We mainly consider $V_{x} \otimes K_{\mathfrak{p}}$ and $V_{k^{\prime}} \otimes K_{\mathfrak{p}}$ (over $K$ ) in this section. It is clear that $W_{p}$ is uniquely extended to the $K_{p}$-linear isomorphism between $V_{\chi} \otimes K_{\mathfrak{p}}$ and $V_{\bar{\chi}} \otimes K_{\mathfrak{p}}$, so we also denote this by $W_{p}$. We define $K_{\mathfrak{p}}$-norms $N_{x}$ and $N_{x}^{\prime}$ on $V_{x} \otimes K_{p}$ as follows: for any $f=\sum_{n=1}^{\infty} a_{n} q^{n} \in V_{\chi} \otimes K_{\mathfrak{p}}, a_{n} \in K_{\mathfrak{p}}$, we define $N_{x}$ and $N_{x}^{\prime}$ by

$$
N_{x}(f)=\sup _{n} \nu\left(a_{n}\right)
$$

and

$$
N_{\bar{\chi}}^{\prime}(f)=N_{\bar{\chi}}\left(f \mid W_{p}\right) \text {. }
$$

Hence by virtue of Proposition 4 of Chap II, $\S 2$ in [10], there exists the following decomposition 


$$
V_{x} \otimes K_{\mathfrak{p}}=V_{1} \oplus \cdots \oplus V_{d_{x}}
$$

of $V_{x} \otimes K_{\mathfrak{p}}$ into a direct sum of subspaces $V_{i}$ of dimension 1 such that

$$
N_{\chi}\left(\sum v_{i}\right)=\sup _{i} N_{\chi}\left(v_{i}\right)
$$

and

$$
N_{x}^{\prime}\left(\sum v_{i}\right)=\sup _{i} N_{x}^{\prime}\left(v_{i}\right),
$$

whenever $v_{i} \in V_{i}$ for $1 \leq i \leq d_{\chi}$ where $d_{\chi}$ denotes the dimension of $V_{\chi} \otimes K_{\mathfrak{p}}$ over $K_{\mathrm{p}}$. Here we may assume that $V_{i}=K_{\mathrm{p}} \cdot v_{i}$ with $v_{i} \in V_{i}$ and $N_{\chi}\left(v_{i}\right)=1$ for $1 \leq i \leq d_{x}$. Changing the order of $i$ suitably, we may assume

$$
\begin{cases}N_{\chi}^{\prime}\left(v_{i}\right)<p^{\alpha /(p-1)} & \text { for } 1 \leq i \leq r_{\chi}, \\ N_{z}^{\prime}\left(v_{i}\right) \geq p^{\alpha /(p-1)} & \text { for } r_{\chi}<i \leq d_{\chi} .\end{cases}
$$

ThEOREM 4.2. The notation being as above, we have

$$
r_{\chi}=\operatorname{dim}_{C} S_{(k / 2)(p+1)-(p-1-\kappa)}(N, \psi) \text {. }
$$

Proof. We use the same idea as in the proof of Theorem (3.4) in [4], so we quote several results from [4]. For any odd Dirichlet character $\eta$ $\bmod p$, let

$$
\begin{aligned}
& E_{1, \eta}=1+c_{\eta} \sum_{\substack{m>0 \\
m_{1}>0}} \eta(m) e^{2 \pi i m m_{1} z}, \\
& c_{\eta}=-\frac{2 p}{\sum_{1 \leq a \leq p-1} \eta(a) a}
\end{aligned}
$$

denote the normalized Eisenstein series of type $(1, \eta)$ on $\Gamma_{0}(p)$. Then we have

$$
E_{1, \bar{\omega}} \mid\left[\begin{array}{rr}
0 & -1 \\
p & 0
\end{array}\right]=\frac{c_{\bar{\omega}}}{c_{\omega}} \frac{\sqrt{p}}{C(\omega)} E_{1, \omega}
$$

where $C(\omega)=\sum_{a=1}^{p-1} \omega(a) e^{2 \pi i(a / p)}$ is the Gauss sum.

For each $v_{i}, 1 \leq i \leq d_{x}$, put

$$
h_{i}=v_{i}\left(E_{1, \bar{\omega}}\right)^{(k / 2)(p-1)-(p-1-k)} .
$$

Then $h_{i}$ is written by a $K_{p}$-linear sum of elements in $S_{k_{1}}(N p, \psi)$. Hence we can define

$$
T_{r}\left(h_{i}\right)=h_{i}+\psi(p)^{-1} p^{1-\left(k_{1} / 2\right)}\left(h_{i} \mid W_{p}\right) \mid U(p),
$$

as in [4]. 
Then $T_{r}\left(h_{i}\right)$ is written by a $K_{p}$-linear sum of elements in $S_{k_{1}}(N, \psi)$. We shall prove

$$
T_{r}\left(h_{i}\right) \equiv h_{i}(\bmod \mathfrak{p})
$$

for $i, 1 \leq i \leq r_{x}$.

For any element $f=\sum_{n=1}^{\infty} a_{n} q^{n} \in K_{p}[[q]]$, we define

$$
N(f)=\sup _{n} \nu\left(a_{n}\right) .
$$

Then, in order to prove (4.7), it sufficient to prove

$$
N\left(p^{1-\left(k_{1} / 2\right)} h_{i} \mid W_{p}\right)<1
$$

for $1 \leq i \leq r_{x}$. It is clear that

$$
\begin{aligned}
h_{i} \mid W_{p} & =\left(v_{i} \mid W_{p}\right)\left(E_{1, \bar{\omega}} \mid\left[\begin{array}{rr}
0 & -1 \\
p & 0
\end{array}\right]\right)^{k_{1}-k} \\
& =\left(v_{i} \mid W_{p}\right) \cdot\left(\frac{\sqrt{p} c_{\bar{\omega}}}{C(\omega) c_{\omega}}\right)^{k_{1}-k} E_{1, \omega}^{k_{1}-k} .
\end{aligned}
$$

Therefore, using Proposition (1.2) in [4], we have

$$
\begin{aligned}
N\left(p^{1-\left(k_{1} / 2\right)} h_{i} \mid W_{p}\right) & =p^{-\left(1-\left(k_{1} / 2\right)\right)} N_{x}^{\prime}\left(v_{i}\right) p^{(-(1 / 2)-(1 / p-1))\left(k_{1}-k\right)} \\
& =p^{-(\delta /(p-1))} N_{x}^{\prime}\left(v_{i}\right) .
\end{aligned}
$$

Hence (4.8) holds for $1 \leq i \leq r_{x}$.

Since $h_{i} \equiv v_{i}(\bmod \mathfrak{p})$, we have

$$
T_{r}\left(h_{i}\right) \equiv v_{i}(\bmod \mathfrak{p})
$$

for $1 \leq i \leq r_{x}$. Hence $\left\{\tilde{v}_{i}, 1 \leq i \leq r_{z}\right\}$ are contained in $\tilde{V}_{k_{1}}$. Since $\left\{\tilde{v}_{i}, 1 \leq\right.$ $\left.i \leq r_{x}\right\}$ are linearly independent over $F$, we have

$$
r_{x} \leq \operatorname{dim}_{C} S_{k_{1}}(N, \psi) .
$$

We apply the same argument to $V_{\bar{x}}$. Let

$$
u_{i}=N_{x}^{\prime}\left(v_{i}\right)^{-1}\left(v_{i} \mid W_{p}\right)
$$

for $1 \leq i \leq d_{x}$. Then $\left\{u_{i}, 1 \leq i \leq d_{x}\right\}$ forms a basis of $V_{\bar{z}} \otimes K_{\mathrm{p}}$. For $\bar{\chi}, k$ changes to $p-1-\kappa$.

Hence we have

$$
N_{x}^{\prime}\left(u_{i}\right)<p^{1-(\kappa /(p-1))}
$$

for $r_{x}+1 \leq i \leq d_{x}$. Therefore we have 


$$
d_{\chi}-r_{\chi} \leq \operatorname{dim}_{C} S_{k_{2}}(N, \psi)
$$

By virtue of Theorem 1.1, we have

$$
d_{\chi}=\operatorname{dim}_{C} S_{k_{1}}(N, \psi)+\operatorname{dim}_{C} S_{k_{2}}(N, \psi) .
$$

Combining (4.9), (4.11) and (4.12), we obtain the proof of Theorem 4.2.

Proof of Theorem 1.2. For each $v_{i}, 1 \leq i \leq d_{x}$, there exists an element $f_{i}$ in $V_{\chi}$ satisfying $v_{i} \equiv f_{i}(\bmod \mathfrak{p})$. As $V_{1, \chi}$ and $V_{2, \chi}$, we can take the spaces spanned by $\left\{f_{i}, 1 \leq i \leq r_{\chi}\right\}$ and $\left\{f_{i}, r_{x}+1 \leq i \leq d_{x}\right\}$ respectively over $K$. Then the proof of Theorem 4.2 shows that (1.12), (1.13) and (1.14) are true.

\section{§5. Proof of Corollary $\mathbf{1 . 3}$}

We use the same notation as in the proof of Theorem 4.2, except for $d_{x}$ and $r_{x}$. We simply write $d$ and $r$ instead of $d_{x}$ and $r_{x}$. Let $\left\{v_{i}, 1 \leq i \leq d\right\}$ be the basis of $V_{x} \otimes K_{p}$. For any positive integer $n$ such that $(n, N p)=1$, we simply denote by $T(n)$ the Hecke operator of degree $n$ acting on $S_{k}(N p, \psi \chi)$ or $S_{k^{\prime}}(N, \chi)$. Since $T(n)$ are $K$-linear endomorphisms of $V_{\mathrm{x}}$ or $V_{k^{\prime}}, T(n)$ are uniquely extended to $K_{\mathrm{p}}$-linear endomorphisms of $V_{\chi} \otimes K_{\mathrm{p}}$ or $V_{k^{\prime}} \otimes K_{\mathrm{p}}$, which we also denote by $T(n)$. Let $\ell$ be a prime such that $\ell \nmid N p$. Then, for any $v=\sum_{n=1}^{\infty} a(n) q^{n} \in V_{\chi} \otimes K_{p}, a(n) \in K_{p}$, we have

$$
v \mid T(\ell)=\sum_{n=1}^{\infty}\left\{a(n \ell)+\ell^{k-1} \psi \chi(\ell) a\left(\frac{n}{\ell}\right)\right\} q^{n},
$$

where $a(n / \ell)=0$ if $\ell \nmid n$.

Theorem 1.2 implies that, for each $v_{i}, 1 \leq i \leq r$, there exists an element $f_{i}$ in $V_{k_{1}} \otimes K_{p}$ such that

$$
v_{i} \equiv f_{i}(\bmod \mathfrak{p}) .
$$

Then it is clear that $\left\{f_{i}, 1 \leq i \leq r\right\}$ forms a basis of $V_{k_{1}} \otimes K_{p}$. For any $f=\sum_{n=1}^{\infty} b(n) q^{n} \in V_{k_{1}} \otimes K_{p}, b(n) \in K_{p}$, we have

$$
f \mid T(\ell)=\sum_{n=1}^{\infty}\left\{b(n \ell)+\ell^{k_{1}-1} \psi(\ell) b\left(\frac{n}{\ell}\right)\right\} q^{n} .
$$

Since $\ell^{k_{1}-1}=\ell^{(k / 2)(p+1)-(p-1-\kappa)-1} \equiv \ell^{k-1} \cdot \ell^{\kappa} \equiv \chi(\ell) \ell^{k-1}(\bmod \mathfrak{p})$, by (5.1), (5.2) and (5.3), we have

$$
v_{i}\left|T(n) \equiv f_{i}\right| T(n) \quad(\bmod \mathfrak{p})
$$

for any $i, 1 \leq i \leq r$ and for any positive integers $n$ such that $(n, N p)=1$. 
We should remark that $V_{k_{1}}$ is closed under the action of $T(n)$, but the space spanned by $v_{i}, 1 \leq i \leq r$ may not be closed under the action of any $T(n)$.

Take suitable constants $c_{i} \in K_{\mathfrak{p}}$ satisfying

$$
\left\{\begin{array}{l}
v_{i}^{\prime}=v_{i} \mid W_{p}, \\
u_{i}=c_{i} v_{i}^{\prime}, \quad N_{\bar{\chi}}\left(u_{i}\right)=1 \quad \text { for } 1 \leq i \leq d .
\end{array}\right.
$$

For any $u=\sum_{n=1}^{\infty} c(n) q^{n} \in V_{\bar{z}} \otimes K_{\mathfrak{p}}, c(n) \in K_{\mathfrak{p}}$, we have

$$
u \mid T(\ell)=\sum_{n=1}^{\infty}\left\{c(n \ell)+\ell^{k-1} \psi(\ell) \bar{\chi}(\ell) c\left(\frac{n}{\ell}\right)\right\} q^{n} .
$$

Theorem 1.2 implies that for each $i, r+1 \leq i \leq d$, there exists an element $g_{i}$ in $V_{k_{2}} \otimes K_{\mathrm{p}}$ such that

$$
u_{i} \equiv g_{i} \quad(\bmod \mathfrak{p}) .
$$

For any $g=\sum_{n=1}^{\infty} d(n) q^{n} \in V_{k_{2}} \otimes K_{\mathfrak{p}}, d(n) \in K_{\mathfrak{p}}$, we have

$$
g \mid T(\ell)=\sum_{n=1}^{\infty}\left\{d(n \ell)+l^{k_{2}-1} \psi(\ell) d\left(\frac{n}{\ell}\right)\right\} q^{n} .
$$

Since $\ell^{k_{2}-1}=\ell^{(k / 2)(p+1)-k-1} \equiv \ell^{k-1} \cdot \ell^{-x} \equiv \ell^{k-1} \bar{\chi}(\ell)(\bmod \mathfrak{p})$, by (5.6), (5.7) and (5.8), we have

$$
u_{i}\left|T(n) \equiv g_{i}\right| T(n) \quad(\bmod \mathfrak{p}),
$$

for any $i, r+1 \leq i \leq d$ and for any positive integer $n$ such that $(n, N p)$ $=1$. Let

$$
\begin{aligned}
& {\left[v_{r+1}, \cdots, v_{d}\right] \mid T(n)=\left[v_{1}, \cdots, v_{d}\right] A(n),} \\
& {\left[u_{r+1}, \cdots, u_{d}\right] \mid T(n)=\left[u_{1}, \cdots, u_{d}\right] C(n),} \\
& {\left[g_{r+1}, \cdots, g_{d}\right] \mid T(n)=\left[g_{r+1}, \cdots, g_{d}\right] D(n),}
\end{aligned}
$$

where $A(n), C(n), D(n)$ are $d \times d-r, d \times d-r, d-r \times d-r$ matrices whose elements are in $\mathfrak{o}_{\mathrm{p}}$. Then (5.9) shows that

$$
\left.C(n)(\bmod \mathfrak{p})=\left[\frac{O}{D(n)}\right]\right\}_{r} \quad(\bmod \mathfrak{p}) .
$$

In [1], Asai proved that, for any positive integer $n$ such that $(n, N p)=1$, it holds

$$
\chi(n)\left\{v_{i} \mid W_{p}\right\}\left|T(n)=\left\{v_{i} \mid T(n)\right\}\right| W_{p},
$$

for any $i, 1 \leq i \leq d$. We should remark that he considered only when 
the level is square free but it is easily shown that his method is applicable to our case.

Therefore we have

$$
\begin{aligned}
{\left[v_{r+1}, \cdots, v_{d}\right]|T(n)| W_{p} } & =\left[v_{1}, \cdots, v_{d}\right] A(n) \mid W_{p} \\
& =\left(\left[v_{1}, \cdots, v_{d}\right] \mid W_{p}\right) A(n) \\
& =\left[u_{1}, \cdots, u_{d}\right]\left[\begin{array}{ccc}
c_{1}^{-1} & 0 \\
& \ddots & \\
0 & & c_{d}^{-1}
\end{array}\right] A(n)
\end{aligned}
$$

and

$$
\begin{aligned}
\chi(n)\left[v_{r+1}, \cdots, v_{d}\right]\left|W_{p}\right| T(n) & =\chi(n)\left[u_{r+1}, \cdots, u_{d}\right]\left[\begin{array}{ccc}
c_{r+1}^{-1} & & 0 \\
& \ddots & \\
0 & & c_{d}^{-1}
\end{array}\right] \mid T(n) \\
& =\chi(n)\left[u_{1}, \cdots, u_{d}\right] C(n)\left[\begin{array}{ccc}
c_{r+1}^{-1} & & 0 \\
& \ddots & \\
0 & & c_{d}^{-1}
\end{array}\right] .
\end{aligned}
$$

Hence we have

$$
\left[\begin{array}{ccc}
c_{1}^{-1} & & 0 \\
& \ddots & \\
0 & & c_{d}^{-1}
\end{array}\right] A(n)=\chi(n) C(n)\left[\begin{array}{ccc}
c_{r+1}^{-1} & & 0 \\
& \ddots & \\
0 & & c_{d}^{-1}
\end{array}\right]
$$

so

$$
\begin{aligned}
& C(n)=\chi(n)^{-1}\left[\begin{array}{ccc}
c_{1}^{-1} & & 0 \\
& \ddots & \\
0 & & c_{d}^{-1}
\end{array}\right] A(n)\left[\begin{array}{ccc}
c_{r+1} & & 0 \\
& \ddots & \\
0 & & c_{d}
\end{array}\right] \\
& =\chi(n)^{-1}\left[\begin{array}{ccc} 
& * & \\
\hline a_{r+1, r+1} & & * \\
& \ddots & \\
* & & a_{d, d}
\end{array}\right]^{\}_{r}}
\end{aligned}
$$

where $A(n)=\left(a_{i j}\right)$.

Then combining (5.4), (5.9), (5.11) and (5.12) we have

(5.13) $\operatorname{tr} T(n) \quad$ on $V_{\chi} \equiv \operatorname{tr} T(n) \quad$ on $V_{k_{1}}+\chi(n) \operatorname{tr} T(n)$ on $V_{k_{2}}(\bmod \mathfrak{p})$.

This completes the proof. 
Remark 5.1. In subsequent papers [6], we shall give another method to prove these congruences. It is to compute $\operatorname{tr} T(n)$ on $V_{x}(\bmod \mathfrak{p})$, $\operatorname{tr} T(n)$ on $V_{k^{\prime}}(\bmod \mathfrak{p})$ directly by using Hijikata's explicit trace formulae for Hecke operators [2], and to compare both hands sides of (5.13).

Remark 5.2. If (5.13) is proved by an another method, we can get simpler proof of Theorem 1.1 applying (5.13) to the case $n=1$.

\section{§6. Supplements to Theorem 1.2}

Here we assume that $p \equiv 1(\bmod 4), k$ is odd, $k \geq 3$ and $\psi \chi(-1)=-1$. In Theorem $3.3, \S 3$, we proved certain identities of dimensions of spaces of cusp forms analogous to Theorem 1.1. Hence it is natural to study a statement analogous to Theorem 1.2 in this case. We slightly modify the proof of Theorem 1.2 at following points:

(i) $K$ must be enlarged to contain $\sqrt{d}$ for all positive divisors $d$ of $N p$.

(ii) We consider $S_{k}\left(N p, \psi \chi \omega^{(p-1) / 2}\right)$ instead of $S_{k}(N p, \psi \chi)$. Since $p \equiv 1$ $(\bmod 4)$, we have $\omega^{(p-1) / 2}(-1)=1$ and $\psi \chi \omega^{(p-1) / 2}(-1)=-1$. Hence the order of $\chi \omega^{(p-1) / 2}$ coincides with that of $\chi$. Therefore we have

$$
\operatorname{dim}_{C} S_{k}\left(N p, \psi \chi \omega^{(p-1) / 2}\right)=\operatorname{dim}_{C} S_{k}(N p, \psi \chi) .
$$

Since $\omega^{(p-1) / 2}$ is a real valued character, we have

$$
\left(\overline{\chi \omega^{(p-1) / 2}}\right)=\bar{\chi} \omega^{(p-1) / 2} \text {. }
$$

(iii) $V_{x}$ should be changed as follows:

$$
V_{\chi}=\left\{f(z)=\sum_{n=1}^{\infty} a_{n} q^{n} \in S_{k}\left(N p, \psi \chi \omega^{(p-1) / 2}\right) \mid a_{n} \in K \text { for all } n \geq 1\right\} .
$$

(iv) Since $k$ is odd, we have

$$
\bar{\omega}^{(p-1)(k / 2)-(p-1-\kappa)}=\bar{\omega}^{(p-1) / 2} \bar{\omega}^{\kappa}=\omega^{(p-1) / 2} \bar{\omega}^{\kappa} .
$$

Hence, following the arguments in $\S 4, \S 5$, we can prove

TheOREm 6.1. The notation being as above, there is a decomposition of $V_{\chi}$ into a direct sum of subspaces $V_{1, \chi}$ and $V_{2, \chi}$ satisfying following properties:

$$
\begin{aligned}
& \operatorname{dim}_{K} V_{1, \chi}=\operatorname{dim}_{C} S_{k_{1}}(N, \psi), \\
& \operatorname{dim}_{K} V_{2, \chi}=\operatorname{dim}_{C} S_{k_{2}}(N, \psi) .
\end{aligned}
$$




$$
\begin{gathered}
\widetilde{V_{1, \chi}}=\tilde{V}_{k_{1}} . \\
\left(\widetilde{V_{2, \chi} \mid W_{p}}\right)=\tilde{V}_{k_{2}} .
\end{gathered}
$$

CoRollaRy 6.2. For any Hecke operator $T(n)$, with $(n, N p)=1$, the following congruence holds:

$$
\begin{aligned}
& \operatorname{tr} T(n) \quad \text { on } S_{k}\left(N p, \psi \times \omega^{(p-1) / 2}\right) \equiv \operatorname{tr} T(n) \text { on } S_{k_{1}}(N, \psi) \\
& +n^{\kappa} \operatorname{tr} T(n) \text { on } S_{k_{2}}(N, \psi)(\bmod p) \text {. }
\end{aligned}
$$

\section{REFERENCES}

[1] T. Asai, On the Fourier coefficients of automorphic forms at various cusps and some applications to Rankin's convolution, J. Math. Soc. Japan, 28 (1976), 48-61.

[2] H. Hijikata, Explicit formula of the traces of Hecke operators for $\Gamma_{0}(N)$, J. Math. Soc. Japan, (1974), 56-82.

[ 3 ] — - On certain identities between the traces of Hecke operators, Proc. Japan Acad., 48 (1972), 585-588.

[4] M. Koike, Congruences between cusp forms and linear representations of the Galois group, Nagoya Math. J., 64 (1976), 63-85.

[5 ] —- A note on modular forms mod p, Proc. Japan Acad., 55 (1979), 313-315.

[6] - Eigenvalues of Hecke operators mod $p$, preprint.

[ 7 ] T. Miyake, On automorphic forms on $G L_{2}$ and Hecke operators, Ann. of Math., 94 (1971), 174-189.

[ 8 ] J.-P. Serre, Formes modulaires et fonctions zêta $p$-adiques, Modular functions of one variable. III. Proc. Intern. Summer School, Univ. Antwerp. Lect. Notes in Math., 350, Springer, (1972) 191-268.

[9] G. Shimura, Introduction to the arithmetic theory of automorphic functions, Iwanami Shoten and Princeton University Press, 1971.

[10] A. Weil, Basic Number Theory, Grundl. Math. Wiss., 144, Berlin-Heidelberg-New York, 1967.

Department of Mathematics

Nagoya University

Chikusa-ku, Nagoya 464

Japan 